\title{
Amiodarone in Pediatric Patients
}

\author{
MACDONALD DICK II, M.D., and WILLIAM A. SCOTT, M.D. \\ From the Division of Pediatric Cardiology, C.S. Mott Children's Hospital, and the Department of Pediatrics, \\ University of Michigan Medical School, Ann Arbor, Michigan
}

\begin{abstract}
DICK M, II, and SCOTT WA: Amiodarone in pediatric patients. The reported world clinical experience of amiodarone in children is reviewed; the known age-dependent electrophysiological and pharmacokinetic characteristics of amiodarone are examined; and guidelines for the use of amiodarone in children are suggested. (Clin. Prog. Electrophysiol. Pacing, Vol. 4, 1986)
\end{abstract}

pharmacokinetics, side effects, supraventricular tachycardia, ventricular fibrillation, ventricular tachycardia.

Recent interest in cardiac arrhythmias in children has been motivated by a growing awareness and understanding of primary rhythm disorders in the young as well as by an increasing emergence of cardiac arrhythmias following surgical repair of many congenital heart defects. ${ }^{1}$ An increased understanding and use of both conventional and investigational antiarrhythmic medications in children has, by necessity, accompanied this clinical development. Of particular interest is the emergence of amiodarone hydrochloride, an iodinated benzofuran derivative, in the treatment of arrhythmias. This article focuses on the electophysiology, pharmacology, experience, side effects, dosages, and indications for amiodarone especially relevant to the pediatric patient.

\section{Electrophysiology and Pharmocology}

The electrophysiological effects and pharmacology of amiodarone are reviewed elsewhere in this issue. Among the observed electrophysiological changes induced by amiodarone are a slowing of the spontaneous sinus node discharge rate, slowing of atrioventricular conduction, and prolongation of atrial and ventricular

Address for reprint: Macdonald Dick II, M.D., F1123, Box 0204,C.S. Mott Children's Hospital, University of Michigan Medical Center, 1500 E. Medical Center Drive, Ann Arbor, MI 48109-0204. effective refractory periods. ${ }^{2}$ Both acute intravenous and chronic oral amiodarone prolong adult human and canine ventricular repolarization (type III effects) ${ }^{3}$ and exhibit rate-related prolongation of the ventricular action potential as well as QRS duration (type I effects) ${ }^{4}$ Recent work by Yabek and associates suggests important age-dependent effects of amiodarone. ${ }^{5}$ Acute data from the neonatal canine ventricle have demonstrated no change in ventricular repolarization, suggesting a decreased sensitivity to amiodarone in the young mammalian myocardium. Further, amiodarone produces a greater concentration-dependent shortening of the action potential and effective refractory period of neonatal canine Purkinje fibers when compared to the effects on the adult canine Purkinje fiber. In contrast, rate-related decreases in the action potential amplitude and $\mathrm{V}_{\max }$ of both neonatal and adult tissues are similar, and thus are not age-dependent. These findings suggest that, when compared to the adult myocardium, type III effects may be less operative in the immature myocardium in which dispersion of refractoriness is minimal. Furthermore, the rate-related type I antiarrhythmic action of amiodarone may be of particular significance in the young in whom both the baseline and tachycardia heart rate tend to be higher. ${ }^{5}$

The pharmacokinetics of orally administered amiodarone are significantly different between 
the adult and child. The time of onset of therapeutic effect is four to five days in the pediatric population compared to nine to ten days in the adult population. ${ }^{6-8}$ Although the elimination of the drug by both groups is biexponential with an initial rapid phase followed by a more prolonged second phase due to clearance from tissue stores, the half-life of this elimination is two to three weeks in children compared to two to four months in the adult, perhaps due to a more rapid equilibration of the drug between plasma and tissue in the young. ${ }^{6-8}$ In both children and adults, amiodarone is metabolized in part by the liver to the nonactive desethylamiodarone; this metabolite and the parent drug are then cleared by the liver. Therapeutic plasma concentrations, considered to be between 1.0 and $2.5 \mu \mathrm{g} / \mathrm{ml}$, correlate only fairly with either beneficial effect or side effects. ${ }^{9,10}$ On the other hand, correlation has been shown between therapeutic antiarrhythmic effect and prolongation of the QT interval in adults receiving 400-600 mg of amiodarone per day. ${ }^{11}$

Information regarding the fetal pharmacology of amiodarone is incomplete. A 27-week gestation fetus with atrial flutter $(260 \mathrm{bpm})$ was treated by oral administration of amiodarone (in conjunction with verapamil) to the mother. The fetal ventricular rate slowed with resolution of fetal heart failure. The fetus progressed to term without adverse outcome; direct current cardioverison to sinus rhythm was achieved at 24 hours of age. ${ }^{12}$ In two other women who were undergoing treatment for arrhythmia in the third trimester, the fetal-to-maternal plasma concentration ratio was 0.2 and 0.25 without adverse side effects. ${ }^{8}$ In another report, the mother was treated with amiodarone $(200 \mathrm{mg}$ per day maintenance dose) for supraventricular tachycardia beginning at the 16 th week. Delivery at 39 weeks of a normal newborn was uneventful. Nine hours after the last maternal dose, the cord blood amiodarone concentration was $0.08 \mu \mathrm{g} / \mathrm{ml}$, the placenta serum concentration, $0.23 \mu \mathrm{g} / \mathrm{ml}$, and the placental tissue concentration, $7.2 \mu \mathrm{g} / \mathrm{g}^{13}$, similar to reported heart tissue concentrations after chronic therapy. ${ }^{8}$ The mother's amiodarone serum level was $0.8 \mu \mathrm{g} / \mathrm{ml}$. Maternal and infant thyroid studies were normal. At two weeks of age the child's amiodarone serum was $0.06 \mu \mathrm{g} / \mathrm{ml}$. The infant's QTc was 0.49 seconds at 24 hours of age, and 0.39 seconds at 7 weeks of age. ${ }^{13}$ Thus the fetal-to-maternal serum concentration ratio in this infant at delivery was approximately 0.1 , demonstrating transplacental passage but no adverse fetal effects after continuous exposure to amiodarone beginning early in the second trimester. This small experience suggests that amiodarone may be safe in the second and third trimester. On the other hand, since there are no reports of first-trimester use, and because of the widespread tissue distribution as well as the multiple organ toxicity in the adult, the possibility of teratogenesis during the first trimester may be great; thus, we suggest that the drug should not be used in early pregnancy, and perhaps only sparingly in later pregnancy.

\section{Clinical Experience}

Amiodarone has been remarkably effective in reducing the rate and frequency of either automatic or reentrant supraventricular and ventricular arrhythmias in children. We were able to find detailed documentation of approximately 200 children reported in the literature (Table I), the vast majority reported by Coumel. ${ }^{6}$ Amiodarone was effective in controlling most atrial arrhythmias. Because of its effect of prolonging the refractory period of the accessory pathway connection as well as decreasing the conduction velocity of the circus movement in different parts of the circuit, ${ }^{14}$ amiodarone is especially useful for control of arrhythmias in patients with the Wolff-Parkinson-White syndrome, ${ }^{15}$ including children. ${ }^{16}$ Coumel $^{6}$ reports fair to good (normal sinus rhythm or a marked decrease in frequency and rate of the tachycardia) in $92 \%$ (86 of 93 ) of children with atrial arrhythmias and $85 \%$ of children with ventricular arrhythmias. Garson ${ }^{7}$ reported complete or partial effectiveness of amiodarone in $96 \%$ of children with atrial flutter, $86 \%$ of children with ventricular tachcardia, and $78 \%$ of patients with supraventricular tachycardia (overall success $87 \%$ ). Among our 7 patients who have received amiodarone (3 with supraventricular tachy- 
TABLE I

\begin{tabular}{|c|c|c|c|c|c|c|c|c|c|c|c|}
\hline & \multicolumn{3}{|c|}{ Atrial } & \multirow[b]{2}{*}{ WPW } & \multirow[b]{2}{*}{ Other ${ }^{*}$} & \multicolumn{2}{|c|}{ Junctional } & \multicolumn{3}{|c|}{ Ventricular } & \multirow[b]{2}{*}{ Total } \\
\hline & Automatic & FIb/flut & sss & & & Reentrant & Automatic & VPB & VT & VF & \\
\hline Coume|6,32 & 43 & 4 & 7 & 8 & 39 & 7 & 7 & 15 & 5 & 0 & 135 \\
\hline Garson $^{7}$ & $1 / 1^{* *}$ & $15 / 16$ & 0 & $1 / 3$ & $2 / 2$ & $1 / 1$ & $2 / 2$ & 0 & $12 / 14$ & 0 & 39 \\
\hline Costigan 25 & 0 & 3 & 0 & 1 & 5 & 0 & 0 & 0 & 6 & 0 & 15 \\
\hline Shahar 16 & 0 & 0 & 0 & $10 / 10$ & 0 & 0 & 0 & 0 & 0 & 0 & 10 \\
\hline Present report & 0 & $1 / 3$ & $3 / 3$ & 0 & 0 & 0 & 0 & 0 & 0 & $1 / 1$ & 7 \\
\hline Pickoff21 & 0 & 0 & 0 & 0 & 0 & 0 & 0 & $1 / 1$ & $3 / 3$ & 0 & 4 \\
\hline Zeevi31 & $1 / 1$ & 0 & 0 & 0 & 0 & 0 & 0 & 0 & 0 & 0 & 1 \\
\hline Strasberg 30 & 0 & 0 & 0 & $1 / 1$ & 0 & 0 & 0 & 0 & 0 & 0 & 1 \\
\hline Rey ${ }^{12}$ & 0 & $1 / 1$ & 0 & 0 & 0 & 0 & 0 & 0 & 0 & 0 & 1 \\
\hline Yagupsky24 & 0 & 0 & 0 & 1 & 0 & 0 & 0 & 0 & 0 & 0 & 1 \\
\hline Total & 45 & 27 & 10 & 24 & 46 & 8 & 9 & 16 & 28 & 1 & 214 \\
\hline
\end{tabular}

-The majority were patients with supraventricular tachycardia, not otherwise specified.

"Where applicable, the first number indicates patients with successful response and the second indicates the total number of patients.

cardia, 2 with atrial fibrillation, 1 with atrial flutter, and 1 with syncope and ventricular fibrillation), all but the 2 patients with atrial fibrillation were improved.

In contrast to prior experience in which junctional ectopic tachcardia was notoriously difficult to treat with any medication and was associated with a significant mortality, ${ }^{17}$ a beneficial response was noted in most patients with junctional arrhythmias. One patient died suddenly with junctional ectopic tachycardia after experiencing initial clinical improvement. ${ }^{?}$

Several authors have reported success in controlling ventricular tachycardia/ventricular fibrillation in $52-69 \%$ of adult patients at $12-14$ months, ${ }^{18-20}$ and $29 \%$ at 24 months. ${ }^{20}$ Experience with ventricular tachycardia in children, due to the different distribution of arrhythmias between adults and children along with the virtual absence of clinical ischemic heart disease in children, is less extensive; accumulated data (Table I) indicate approximately $87 \%$ favorable response to amiodarone in children with ventricular arrhythmias. ${ }^{6,7,21}$

This overall experience is even more impressive in view of the fact that the drug was available only as an investigational agent in the United States until March 1986 and that many of these arrhythmias had failed control with more conventional agents. Thus, selection of these patients, at least in the North American experience, is weighted toward children with intractable, difficult-to-manage arrhythmias.

\section{Side Effects}

The type and frequency of side effects in children are at variance with the adult experience. The latter experience indicates that corneal, cutaneous, gastrointestinal, hepatic, neurological, endocrine, renal, pulmonary, and proarrhythmic side effects are not uncommon. ${ }^{10,15}$ These side effects have been reported in approximately $50 \%$ of patients. $10,18,20,22$ Pulmonary fibrosis, occurring in $1-6 \%$ of adults, 12,20,22 and proarrhythmic effects are the most serious in adults. Neurological complaints including tremors and peripheral neuropathy are frequent in adults, occurring in $50 \% .^{23}$

In contrast to the adult population, most reports (Table II) have cited few significant side effects in children. Neurological symptoms in children may present as alterations in behavior (nightmares or personality changes). ${ }^{4}$ Pulmonary fibrosis is unreported in the young; fatal hepatic failure has been reported. ${ }^{24}$ We have seen proarrhythmic effects (increased ventricular ectopic activity) in a 16 -year-old girl. The patient was successfully managed with both amiodarone and procainamide initially. Procainamide was then gradually withdrawn, with 
TABLE ॥

Side Effects of Amiodarone

\begin{tabular}{|c|c|c|c|c|c|c|}
\hline & Ocular & Cutaneous & Hepatic & Neurological & Thyroid & Arrhythmia \\
\hline Coume| 6,32 & $3 / 135$ & $4 / 135$ & none & $4 / 135$ & $3 / 135$ & none \\
\hline Garson $^{7}$ & $7 / 39$ & $3 / 39$ & none & 2/39 & none & none \\
\hline Costigan 25 & $1 / 17$ & none & none & none & $2 / 17$ & $1 / 17$ \\
\hline Shahar ${ }^{16}$ & $1 / 10$ & $1 / 10$ & none & none & none & none \\
\hline Present report & $1 / 6$ & none & none & none & none & $1 / 6$ \\
\hline Pickoff21 & $2 / 4$ & none & none & none & none & none \\
\hline Zeeviji & none & none & none & none & none & none \\
\hline Strasberg 30 & none & none & none & none & none & none \\
\hline Rey ${ }^{12}$ & none & none & none & none & none & none \\
\hline Yagupsky24 & none & none & $1 / 1$ & none & none & none \\
\hline Total (percentage) & 6.54 & 3.0 & 0.5 & 3.0 & 2.0 & 0.7 \\
\hline
\end{tabular}

successful suppression of both her supraventricular and ventricular arrhythmias with amiodarone alone. Children with side effects tend to be older ( $\geq 10$ years of age) (6,7 $^{6}$ as well as to receive a higher dose ( $\geq 400 \mathrm{mg}$ per day). In only 12 of 215 children ( $9 \%$ ) was amiodarone withdrawn specifically because of adverse effects. A reverse $T 3$ level $\geq 90 \mathrm{ng} / \mathrm{dL}$ has been associated with significant side effects in children. ${ }^{7}$

The one major exception with regard to side effects in the child may be hypothyroidism, exemplified by the report of Costigan and associates. ${ }^{25}$ These investigators prospectively studied 15 children receiving amiodarone. Three infants (11-41 months) developed either clinical or biochemical hypothyroidism; two required treatment. These three children, because of the severity of the arrhythymia, required the highest dosage per kilogram per day to control their arrhythmia. This incidence (3 of 15 ) is higher than appreciated in previous reports in children, and underscores, by the clustering in the 3-41-month-old child, the importance of monitoring thyroid function, including reverse $\mathrm{T}$, in the young receiving amiodarone. Although more of a direct and predictable effect on atrioventricular conduction than a side effect, 5 of the 214 patients required pacemaker implantation because of deterioration in atrioventricular conduction. ${ }^{6,7,25}$

An adverse interaction with other drugs may occur. Digoxin levels are increased while a child is taking amiodarone due to reduced renal clearance of digoxin resulting from decreased digoxin secretion as well as an amiodaroneinduced decrease in the volume of distribution. The child should have his digoxin dose reduced by one-half if he or she receives amiodarone concomitantly. Further, there is a high risk of toxic interaction with many type I antiarrhythmia agents; bradycardia and hypotension have been reported with concomitant use of beta blocking and calcium channel blocking agents. ${ }^{27}$ Finally, amiodarone has been shown to potentiate the effects of warfarin. ${ }^{27,28}$ In general, side effects appear to be less frequent and less severe in the young; nonetheless, because of the unknown long-term effects of amiodarone in the growing child, the development of possible side effects must be carefully monitored.

\section{Dosage}

The dosage of amiodarone varies widely; loading doses will vary from $10-60 \mathrm{mg} / \mathrm{kg} /$ day for 3-5 days with maintenance doses varying from $5-27 \mathrm{mg} / \mathrm{kg} /$ day. In general, most patients are loaded with the lower dose range and can then be maintained on $5-10 \mathrm{mg} / \mathrm{kg} /$ day. ${ }^{7,25}$ Supraventricular tachycardia generally requires a lower dose for successful response than does ventricular tachycardia. Because serious side effects are dose-related, efforts to reduce the dose to minimal levels that control the arrhythmia are desirable. Therapeutic and toxic responses can be monitored by measuring serum reverse $\mathrm{T} 3$ concentrations. ${ }^{7,29}$ Although some children have received amiodarone for as long 
as six years, ${ }^{6}$ more long-term (more than one year) experience in children is limited.

\section{Indications and Conclusion}

Amiodarone has many of the attributes of the ideal universal antiarrhythmic agent. It has a long half-life, can be administered once a day, and often less frequently, is highly effective, especially in supraventricular tachycardia, and appears to be well tolerated in children. Nonetheless, the toxicity, although considerably less in terms of lethal dose by weight compared to other antiarrhythmic agents, involves virtually every organ system. Its effects on the growing child are unknown and should be considered potentially very serious. Thus, amiodarone should be reserved for the child with life-threatening and/or cardiac disabling arrhythmias only after other forms of treatment have been exhausted. Although the drug has been released in the United States by the FDA, its use is confined to adults with life-threatening ventricular arrhythmias. Thus, its use in children should be reserved for clearly life-threatening or intractable disabling arrhythmias until further data regarding its effect on human development are available.

\section{References}

1. Dick, M., and Campbell, R.M.: Advances in the management of cardiac arrhythmias in children. Pediatr. Clin. North Am., 31:1175, 1984.

2. Singh, B.N., and Vaughn Williams, E.M.,: The effects of amiodarone, a new anti-anginal drug, on cardiac muscle. Br. J. Pharmacol., 39:657, 1970.

3. Tuna, I.C., Qi, A., Gornick, C., et al.: Kinetics of electrophysiologic changes during oral loading of amiodarone and after withdrawal of amiodarone in the unsedated dog. Circulation, 72:1380, 1985.

4. Morady, F., DiCarlo, L.A., Krol, R.B., et al.: Acute and chronic effects of amiodarone in ventricular refractoriness, interventricular conduction and ventricular tachycardia induction. J. Am. Coll. Cardiol., 7:148, 1986.

5. Yabek, S.M., Kato, R., and Singh, B.N.: Acute effects of amiodarone on the electrophysiologic properties of isolated neonatal and adult cardiac fibers. J. Am. Coll. Cardiol., 5:1109, 1985.
6. Coumel, P., and Fidelle, J.: Amiodarone in the treatment of cardiac arrhythmias in children: One hundred thirty-five cases. Am. Heart J., 100:1063, 1980.

7. Garson, Jr., A., Gillette, P.C., and McVey, P.: Amiodarone treatment of critical arrhythmias in children and young adults. J. Am. Coll. Cardiol., 4:749, 1984.

8. Latini, R, Tognoni, G., and Kates, R.E.: Clinical pharmacokinetics of amiodarone. Clin. Pharmacokinetics, 9:136, 1984.

9. Rotmensch, H.H., Belhassen, B., Swanson, B.N., et al.: Steady-state serum amiodarone concentrations: Relationships with antiarrhythmic efficacy and toxicity. Ann. Intern. Med., 10:462, 1984.

10. Raeder, E.A., Podrid, P.J., and Lown, B.: Side effects and complications of amiodarone therapy. Am. Heart J., 109:975, 1985.

11. Torres, V., Tepper, D., Flowers, D., et al.: QT prolongation and the antiarrhythmic efficacy of amiodarone. J. Am. Coll. Cardiol., 7:142, 1986.

12. Rey, E. Duperron, L., Gauthier, R., et al.: Transplacental treatment of tachycardia-induced fetal heart failure with verapamil and amiodarone: A case report. Am. J. Obstet. Gynecol., 153:311, 1985.

13. Penn, I.M., Barrett, P.A., Pannikote, V., et al.: Amiodarone in pregnancy. Am. J. Cardiol., 56:196, 1985.

14. Wellens, H.J.J., Lie, K.I., Bar, F.W., et al.: Effect of amiodarone in the Wolff-Parkinson-White syndrome. Am. J. Cardiol., 38:189, 1976.

15. Rosenbaum, M.B., Chiale, P.A., Halpern, M.S., et al.: Clinical efficacy of amiodarone as an antiarrhythmic agent. Am. J. Cardiol., 58:934, 1976.

16. Shahar, E., Barzilay, Z., Frand, M., et al.: Amiodarone in control of sustained tachyarrhythmias in children with WolffParkinson-White syndrome. Pediatrics, 72:813, 1983.

17. Garson, Jr., A., and Gillette P.C.: Junctional ectopic tachycardia in children: Electrocardiography, electrophysiology and pharmacologic response. Am. J. Cardiol., 44:298, 1979.

18. Morady, F., Sauve, M.J., Malone, P., et al.: Longterm efficacy and toxicity of high-dose amiodarone therapy for ventricular tachycardia or ventricular fibrillation. Am. J. Cardiol., 52:975, 1983.

19. Heger, J.J., Prystowsky, E.N., Jackman, W.M., et al.: Amiodarone: Clinical efficacy and electrophysiology during long-term therapy for recurrent ventricular tachycardia or ventricular 
fibrillation. N. Engl. J. Med., 305:539, 1981.

20. Fogoros, R.N., Anderson, K.P., Winkle, R.A., et al.: Amiodarone: Clinical efficacy and toxicity in 96 patients with recurrent, drug-refractory arrhythmias. Circulation, 68:88, 1983.

21. Pickoff, A.S., Singh, S., Flinn, C.J., et al.: Use of amiodarone in the therapy of primary ventricular arrhythmias in children. Dev. Pharmacol. Ther., 6:73, 1983.

22. Smith, W.M., Lubbe, W.F., Whitlock, R.M., et al.: Long-term tolerance of amiodarone treatment for cardiac arrhythmias. Am. J. Cardiol., 57:1288, 1986.

23. Charness, M.E., Morady, F., Scheinman, M.M.: Frequent neurologic toxicity associated with amiodarone therapy. Neurology, 669, 1984.

24. Yagupsky, P., Gonzala, E., Sofer, S., et al.: Fatal hepatic failure and encephalopathy associated with amiodarone. J. Pediatr., 107:967, 1985.

25. Costigan, D.C., Holland, F.J., Daneman, D., et al.: Amiodarone therapy effects on childhood thyroid function. Pediatrics, 77:703, 1986.

26. Koren, G., Hesslein, P.S., and MacLeod, S.M.:
Digoxin toxicity associated wth amiodarone therapy in children. J. Pediatr., 104:467, 1984.

27. Marcus, F.I.: Drug interactions with amiodarone. Am. Heart J., 106:924, 1983.

28. Hamer, A., Peter, T., Mandel, W.J., et al.: The potentiation of warfarin anticoagulation by amiodarone. Circulation, 65:1025, 1982.

29. Nademanee, K., Singh, B.N., Hendrickson, J.A., et al.: Pharmacokinetic significance of serum reverse $T_{3}$ levels during amiodarone treatment: $A$ potential method for monitoring chronic drug therapy. Circulation, 66:202, 1982.

30. Strasberg, B., Davidson, E., and Berand, M.: Amiodarone-induced sinotrial block. Int. J. Cardiol., 8:214, 1985.

31. Zeevi, B., Berant, M., Sclarovsky, S., et al.: Treatment of multifocal atrial tachycardia with amiodarone in a child with congenital heart disease. Am. J. Cardiol., 57:344, 1986.

32. Coumel, P., Lucet, V., and Ngoc, D.D.: The use of amiodarone in children. PACE, 6:930, 1983. 
This document is a scanned copy of a printed document. No warranty is given about the accuracy of the copy. Users should refer to the original published version of the material. 